(1)

CrossMark

\title{
New evidence on the chemoprevention of inhaled steroids and the risk of lung cancer in COPD
}

\author{
Luis M. Seijo (1) ${ }^{1,2}$, Joan B. Soriano $\mathbb{1}^{2,3}$ and Germán Peces-Barba ${ }^{2,4}$
}

Affiliations: ${ }^{1}$ Clínica Universidad de Navarra, Madrid, Spain. ${ }^{2}$ Centro de Investigación Biomédica en Red de Enfermedades Respiratorias, CIBERES, Instituto de Salud Carlos III, Madrid, Spain. ${ }^{3}$ Hospital Universitario La Princesa, Madrid, Spain. ${ }^{4}$ IIS - Fundación Jiménez Díaz, Madrid, Spain.

Correspondence: Luis M. Seijo, Pulmonary Dept, Clínica Universidad de Navarra, Calle Marquesado de Sta. Marta, 3, 28022 Madrid, Spain. E-mail: Iseijodunav.es

@ERSpublications

New evidence regarding the protective effect of inhaled steroids in patients with COPD suggests that a controlled clinical trial is needed to investigate the potential benefit of inhaled steroids in preventing lung cancer in patients with COPD http://bit.ly/2PTBTCO

Cite this article as: Seijo LM, Soriano JB, Peces-Barba G. New evidence on the chemoprevention of inhaled steroids and the risk of lung cancer in COPD. Eur Respir J 2019; 53: 1900717 [https://doi.org/10.1183/ 13993003.00717-2019].

Not long ago, chronic obstructive pulmonary disease (COPD) was considered a Cinderella, an unappealing disease lacking therapeutic options. It was also stigmatised as a consequence of the smoking habit that is a leading cause of COPD worldwide [1]. However, as the Global Initiative for Chronic Obstructive Lung Disease (GOLD) [2], and the joint American Thoracic Society/European Respiratory Society consensus documents suggest [3], COPD should be viewed as treatable and preventable, offering multiple opportunities for disease modification.

Inhalers developed for the treatment of asthma were tested empirically in COPD, long before a mechanistic rationale justified their use. Airway delivery was a common sense solution for a disease characterised by airway inflammation and bronchospasm. Inhaled corticosteroids (ICS) were an obvious therapeutic choice, especially in combination with long-acting bronchodilators (long-acting $\beta$-agonists $(\mathrm{LABA})$ ). Despite the growing evidence in favour of this strategy as a cornerstone of COPD treatment, the role of ICS continues to be controversial in current clinical practice.

The potential benefit of ICS in the prevention of lung cancer is a recurrent argument in this controversy, since smoking-induced inflammation also plays a role in lung carcinogenesis [4]. The advent of combined long-acting bronchodilators and fears of ICS related long-term side-effects, have led to a questioning of the role of ICS in COPD. If lung cancer prevention is a collateral benefit of ICS treatment in COPD, current guidelines may be subject to change. Existing combinations of LABA and ICS are recommended for patients with asthma and COPD overlap, those with moderate or severe obstruction, and patients with frequent exacerbations [2]. These COPD phenotypes benefit from a reduction in eosinophil counts, T-lymphocytes, neutrophils, and other biomarkers $[5,6]$. However, it is unclear if the anti-inflammatory effect can be credited for a reduction in exacerbations or lung cancer incidence.

In this issue of the European Respiratory Journal, the study by RAYMAKERS et al. [7] contributes to a growing body of scientific evidence in favour of lung cancer chemoprevention with ICS. RAYMAKERS et al. [7] analysed 
a large population-based cohort of COPD patients and found that exposure to ICS in a sample of almost 40000 individuals was associated with a 30\% reduction in lung cancer risk (HR 0.70 (95\% CI 0.61-0.80)). Their conclusion persisted after using five different time-dependent methods of quantifying exposure. The $2.5 \%$ incidence of lung cancer in the cohort identified from administrative data in British Columbia, Canada, during a mean follow-up of 5 years, matches that seen in other COPD population-based studies [8].

Keeping in mind that lung cancer is not only more common in patients with COPD, but is also more aggressive and lethal, COPD patients stand to benefit the most from ICS treatment $[9,10]$. The limited evidence in favour of ICS chemoprevention for lung cancer comes from animal studies as well as population-based human studies [11-14]. Prospective evidence, however, is scant and equivocal. Animal trials employing both fluticasone and budesonide seem to point to a protective effect [12, 13]. A sub-study of the COSMOS lung cancer screening trial randomised 200 patients to receive budesonide or placebo for 1 year [14]; after 5 years of follow-up, non-solid and semi-solid nodules diminished in size in patients receiving steroids, despite no change in the incidence of lung cancer or the appearance of new solid nodules. That finding is remarkable given the small sample size and limited therapeutic exposure to ICS. Another study combining various trials of inhaled steroids in COPD concluded that ICS reduce all-cause mortality [10]. In that study, ICS exposure was associated with a trend in favour of diminished cancer mortality. The beneficial effect appears to be dose-dependent, and perhaps more noticeable in women and former smokers $[10,15]$. It has also been reported in asthmatic patients [16].

The current study is unique in implementing a 1-year latency period. This strategy is ingenious, since it avoids potential pitfalls related to the timing of ICS treatment. Ignoring the preclinical phase of lung cancer can otherwise lead to underestimating the potential benefit of ICS. One wonders if 12 months is long enough, since data from the NLST and PLCO trials suggest that the preclinical phase of lung cancer from resectable stage Ia to metastatic disease may last between 3 and 5 years [17]. The authors partially address this limitation by performing a sensitivity analysis with a 2-year latency period, which also yielded statistically significant results.

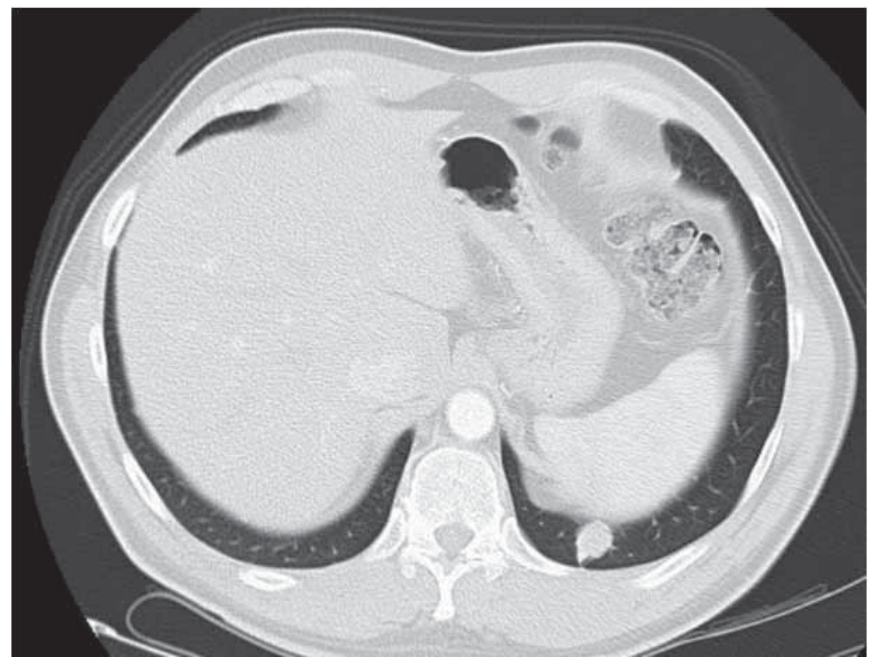

\begin{tabular}{|c|c|c|c|c|c|}
\hline \multirow[b]{2}{*}{ Spirometry } & \multirow[b]{2}{*}{ Reference } & \multicolumn{2}{|c|}{ Pre-bronchodilator } & \multicolumn{2}{|c|}{ Post-bronchodilator } \\
\hline & & Best & $\%$ Reference & Best & $\%$ Reference \\
\hline FVC L & 5.04 & 6.29 & 125 & 6.25 & 124 \\
\hline FEV 1 L & 4.04 & 4.25 & 105 & 4.33 & 107 \\
\hline $\mathrm{FEV}_{1} / \mathrm{FVC} \%$ & 78 & 68 & & 69 & \\
\hline FEF $25-75 \% L \cdot s^{-1}$ & 4.18 & 2.79 & 67 & 2.88 & 69 \\
\hline$P E F L \cdot S^{-1}$ & 9.40 & 9.58 & 102 & 11.01 & 117 \\
\hline
\end{tabular}

FIGURE 1 Computed tomography imaging and spirometry (pre- and post-bronchodilator) of a 52-year-old patient with a left lower lobe pT2aNOMO lung adenocarcinoma. Inhaled steroids are not indicated in this patient population given the mild degree of obstruction and the absence of frequent exacerbations. However, lung cancer may be more common in patients with mild chronic obstructive pulmonary disease (COPD), such as the one pictured here. If inhaled steroids are shown to be effective in preventing lung cancer in patients with COPD, should we reconsider the indication in these patients? FVC: forced vital capacity; FEV1: forced expiratory volume in $1 \mathrm{~s}$; FEF25-75\%: forced expiratory flow at 25-75\% of FVC; PEF: peak expiratory flow. 
Unfortunately, the lack of key variables, such as tobacco exposure, severity of the airway obstruction, a family history of lung cancer or the prevalence of radiological emphysema in the cohort, are obvious limitations of the study. In addition, some authors have found that lung cancer is more likely in patients with mild COPD, who might not benefit from the study's findings since they do not meet current indications for ICS treatment (figure 1) [4]. Furthermore, since COPD is universally underdiagnosed, even in patients with lung cancer, the future of any chemopreventive role for ICS would depend on redoubling efforts to identify and treat COPD in the general population $[18,19]$. In this respect, a diagnosis of COPD based on prescriptions can lead to major biases, including overlap with asthma and even overdiagnosis of COPD. Furthermore, previous population-based studies point to an even lower risk of lung cancer in patients with COPD treated with ICS. The reasons for this discrepancy may be methodological, but lead to the logical conclusion that a well-designed prospective randomised study controlled by placebo is needed. Such a study would allow head-to-head comparisons between placebo and ICS, controlling for COPD stage, smoking history, and potential biases such as the presence and severity of underlying emphysema. It would also clarify the role of ICS in men and women, and in former and active smokers, as well as dosing concerns. In our opinion, the study should include a screening arm capable of studying important surrogate end-points such as airway thickness, emphysema, and the evolution of pulmonary nodules, including ground-glass opacities, solid and semi-solid nodules detected by low dose computed tomography. Alternatively, a re-examination of data from recent clinical trials randomising patients with COPD to combination therapies with and without ICS might be a good start in order to build a case in favour of a larger multinational effort in this regard.

Conflict of interest: L.M. Seijo reports grants from Menarini (funding for lung cancer screening), personal fees from Esteve (speaker fees for a symposium on lung cancer and COPD), personal fees from Roche (speaker fees on lung cancer), personal fees from Medtronic (advisory board fees for speaking on lung cancer screening), travel grants from Chiesi, personal fees from AstraZeneca (speaking fees), personal fees from Suministros Hospitalarios (indwelling pleural catheter symposium), outside the submitted work. J.B. Soriano participated in speaking activities, advisory committees and consultancies during the period 2014-2019 sponsored by: Almirall, AstraZeneca, Boehringer Ingelheim, CHEST, Chiesi, ERS, GEBRO, Grifols, GSK, Linde, Lipopharma, Mundipharma, Novartis, Pfizer, RiRL, Rovi, Sandoz, SEPAR and Takeda. J.B. Soriano declares not receiving ever, directly or indirectly, funding from the tobacco industry or its affiliates. G. Peces-Barba reports grants from GSK (advisory boards, institutional educational programme and research project), Boehringer Ingelheim (institutional educational program), and Chiesi (institutional educational program), outside the submitted work.

\section{References}

Agusti AGN. El sindrome de Lazaro o el revivir de la EPOC. Arch Bronconeumol 2001; 37: 3-6.

2 Vogelmeier CF, Criner GJ, Martinez FJ, et al. Global Strategy for the Diagnosis, Management, and Prevention of Chronic Obstructive Lung Disease 2017 Report: GOLD Executive Summary. Eur Respir J 2017; 49: 1700214.

3 Celli BR, MacNee W, ATS/ERS Task Force. Standards for the diagnosis and treatment of patients with COPD: a summary of the ATS/ERS position paper. Eur Respir J 2004; 23: 932-946.

4 Seijo LM, Zulueta JJ. Understanding the links between lung cancer, COPD, and emphysema: a key to more effective treatment and screening. Oncology 2017; 31: 93-102.

5 Culpitt SV, Maziak W, Loukidis S, et al. Effect of high dose inhaled steroid on cells, cytokines, and proteases in induced sputum in chronic obstructive pulmonary disease. Am J Respir Crit Care Med 1999; 160: 1635-1639.

6 Sandelin M, Mindus S, Thuresson M, et al. Factors associated with lung cancer in COPD patients. Int J Chron Obstruct Pulmon Dis 2018; 13: 1833-1839.

7 Raymakers AJN, Sadatsafavi M, Sin DD, et al. Inhaled corticosteroids and the risk of lung cancer in COPD: a population-based cohort study. Eur Respir J 2019; 53: 1801257.

8 De Torres JP, Marín JM, Casanova C, et al. Lung cancer in patients with chronic obstructive pulmonary diseaseincidence and predicting factors. Am J Respir Crit Care Med 2011; 184: 913-919.

9 Gao YH, Guan WJ, Liu Q, et al. Impact of COPD and emphysema on survival of patients with lung cancer: a meta-analysis of observational studies. Respirology 2016; 21: 269-279.

10 Sin DD, Wu L, Anderson JA, et al. Inhaled corticosteroids and mortality in chronic obstructive pulmonary disease. Thorax 2005; 60: 992-997.

11 Parimon T, Chien JW, Bryson CL, et al. Inhaled corticosteroids and risk of lung cancer among patients with chronic obstructive pulmonary disease. Am J Respir Crit Care Med 2007; 175: 712-719.

12 Witschi H. Successful and not so successful chemoprevention of tobacco smoke-induced lung tumors. Exp Lung Res 2000; 26: 743-755.

13 Wattenberg LW, Wiedmann TS, Estensen RD, et al. Chemoprevention of pulmonary carcinogenesis by brief exposures to aerosolized budesonide or beclomethasone dipropionate and by the combination of aerosolized budesonide and dietary myo-inositol. Carcinogenesis 2000; 21: 179-182.

14 Veronesi G, Lazzeroni M, Szabo E, et al. Long-term effects of inhaled budesonide on screening-detected lung nodules. Ann Oncol 2015; 26: 1025-1030.

15 Liu S-F, Kuo H-C, Lin M-C. Inhaled corticosteroids have a protective effect against lung cancer in female patients with chronic obstructive pulmonary disease: a nationwide population-based cohort study. Oncotarget 2017; 8: 29711-29721.

16 Wang IJ, Liang WM, Wu TN, et al. Inhaled corticosteroids may prevent lung cancer in asthma patients. Ann Thorac Med 2018; 13: 156-162.

17 ten Haaf K, van Rosmalen J, de Koning HJ. Lung cancer detectability by test, histology, stage and gender: estimates from the NLST and the PLCO trials. Cancer Epidemiol Biomarkers Prev 2015; 24: 154-161. 
18 Soriano JB, Zielinski J, Price D. Screening for and early detection of chronic obstructive pulmonary disease. Lancet 2009; 374: 721-732.

19 Collar D P, Guerra M P, Rodriguez P, et al. COPD is commonly underdiagnosed in patients with lung cancer: results from the RECOIL study (retrospective study of COPD infradiagnosis in lung cancer). Int J Chron Obstruct Pulmon Dis 2017; 12: 1033-1038. 